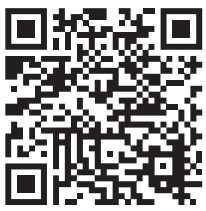
COVID-19, neumonía intersticial, ultrasonido pulmonar.

Keywords: COVID-19, interstitial pneumonia, pulmonary ultrasound.

* Unidad Coronaria, Instituto Nacional de Cardiología «Ignacio Chávez», Secretaría de Salud. Ciudad de México, México.

${ }^{\ddagger}$ Cardiology

Department, Montefiore Hospital and Medical Center, Albert Einstein College of MedicineYeshiva University. Nueva York, EUA. $\S_{\text {Servicio de }}$ Emergencias, Hospital «Dr. Rafael Ángel Calderón Guardia», Caja Costarricense del Seguro Social y World Interactive Network Focused on Critical Ultrasound. San José, Costa Rica. " Terapia Intensiva Postquirúrgica, Instituto Nacional de Cardiología «Ignacio Chávez», Secretaría de Salud. Ciudad de México, México.

Recibido: 20/04/2020

Aceptado:

27/04/2020

\title{
Uso de ultrasonido pulmonar para la detección de neumonía intersticial en la COVID-19
}

\author{
Lung ultrasound for the identification of COVID-19 pneumonia
}

\author{
Rodrigo Gopar-Nieto, ${ }^{*}$ Mercedes Rivas-Lasarte, ${ }^{\ddagger}$ Alejandro Moya-Álvarez, ${ }^{\S}$ \\ Edgar García-Cruz, Daniel Manzur-Sandoval," Alexandra Arias-Mendoza, ${ }^{*}$ \\ Daniel Sierra-Lara Martínez, ${ }^{*}$ Diego Araiza-Garaygordobil*
}

\section{RESUMEN}

La infección por SARS-CoV-2 tiene como manifestación clínica la enfermedad conocida como COVID-19. Si bien el conocimiento de la naturaleza de la enfermedad es dinámico, puesto que se publican cada día decenas de artículos científicos sobre nuevas características de COVID-19,1 la presentación típica es la de neumonía intersticial.2 A pesar de la gran cantidad de información que se ha desarrollado en las últimas semanas, se ha estimado que esta enfermedad puede llegar a tener hasta $72 \%$ de infradiagnóstico, 3 por lo que se requieren herramientas clínicas que sean simples, de fácil acceso, que incrementen la detección de casos de forma factible y que arrojen información con valor pronóstico. Ante esta necesidad, han surgido algunas propuestas para poder realizar el diagnóstico, seguimiento y respuesta al tratamiento de los pacientes con COVID-19, tales como el ultrasonido pulmonar (USP). Cabe mencionar que el USP ha probado ser una técnica eficiente y de fácil reproducibilidad para diagnosticar insuficiencia cardiaca y patologías pleuropulmonares, sobre todo en pacientes críticamente enfermos.4-7 La evidencia de la utilidad de USP en COVID-19 es aún escasa, aunque de forma preliminar parece ser una técnica sensible cuyos hallazgos tienen un elevado estándar de oro. En esta breve revisión haremos énfasis en sus aspectos técnicos, las ventajas y desventajas, y por último una propuesta para el abordaje en este tipo de pacientes.

\section{ABSTRACT}

The SARS-CoV-2 infection has as a clinical manifestation the disease known as COVID-19. Although knowledge of the nature of the disease is dynamic, with dozens of scientific articles being published every day about new features of COVID-19, ${ }^{1}$ the typical presentation is that of interstitial pneumonia. ${ }^{2}$ Despite the large amount of information that has been developed in recent weeks, it has been estimated that this disease can have up to $72 \%$ underdiagnosis, ${ }^{3}$ which requires clinical tools that are simple, easily accessible, and increase the detection of cases in a feasible way and that yield information with prognostic value. Given this need, some proposals have emerged to be able to diagnose, monitor and respond to the treatment of patients with COVID-19, such as pulmonary ultrasound (USP). It is worth mentioning that the USP has proven to be an efficient and easily reproducible technique for diagnosing heart failure and pleuro-pulmonary pathologies, especially in critically ill patients. ${ }^{4-7}$ Evidence of the usefulness of USP in COVID-19 is still scarce, although preliminary, it seems to be a sensitive technique whose findings have a high gold standard. In this brief review we will emphasize its technical aspects, the advantages and disadvantages, and finally a proposal for the approach in this type of patient.

\section{ASPECTOS TÉCNICOS}

$\mathrm{P}$ ara obtener imágenes por este método se puede colocar el transductor de manera perpendicular a la pared torácica, o bien si se quiere obtener imágenes más nítidas de la pleura, se puede colocar de manera paralela a las costillas. Es esencial seguir un orden de exploración estándar para la consistencia del reporte entre operadores (Figura 1). Los transductores más recomendados para este método son los de 3.5 a $5 \mathrm{mHz}$, ya que permiten tener una buena definición de las estructuras $y$ alteraciones que se pueden encontrar. ${ }^{6}$ Por último, cabe mencionar que para este estudio se pueden utilizar transductores lineales, sobre todo para tener una adecuada definición de la pleura y estructuras más superficiales, o sectoriales, para poder visualizar estructuras o artefactos más profundos.

De forma general, un pulmón sano está compuesto en su mayor parte de aire. Cuando 
los ultrasonidos penetran en éste, la mayor parte de ellos son reflejados de nuevo hacia el transductor debido a la gran diferencia de densidad entre la pared pulmonar y la pleura y el parénquima aireado. Esto da lugar a un artefacto horizontal, las líneas A, que de forma indirecta indican que el pulmón no está ocupado. Al contrario, a medida que baja el contenido del aire en el pulmón y que es sustituido por agua u otro componente biológico, aparece un artefacto vertical en cola de cometa, las líneas B. Estas líneas B pueden llegar a ser coalescentes y dar una imagen de pulmón blanco. Además, el USP nos permite visualizar áreas de neumotórax cuando se pierde el deslizamiento pleural, el derrame pleural y la consolidación pulmonar o atelectasias. ${ }^{8}$

\section{HALLAZGOS DEL ULTRASONIDO PULMONAR EN LA COVID-19}

Si bien la literatura es escasa, los hallazgos reportados incluyen engrosamiento pleural, pleura irregular, patrón de líneas B (Figura 1) confluentes y en vidrio despulido, consolidados basales y finalmente en algunos casos derrame pleural. ${ }^{7-10}$

Sin embargo, aún se desconoce el punto de corte de líneas B, o la confluencia de éstas

Figura 1:

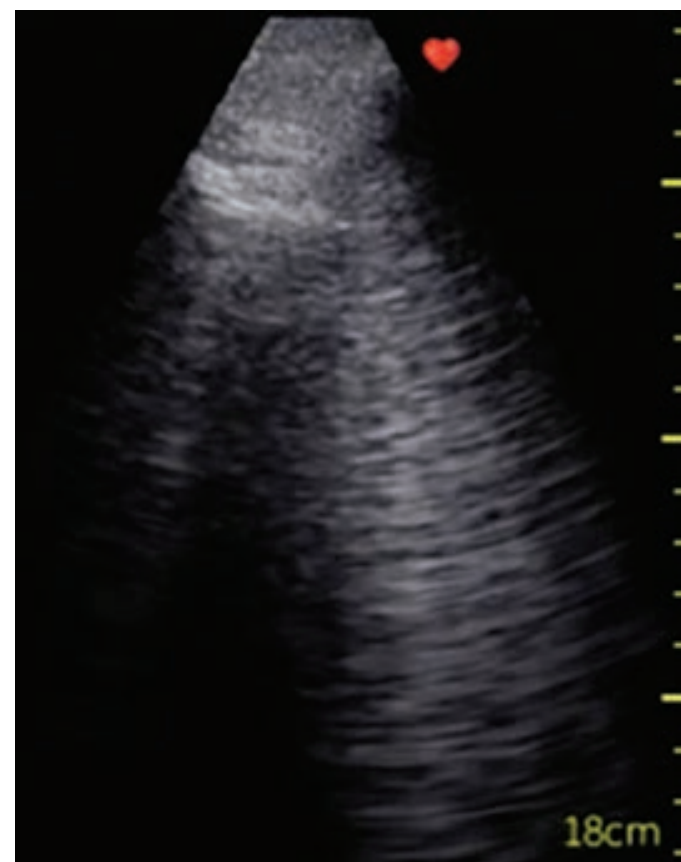

para la predicción de desenlaces, así como características que permitan el diagnóstico diferencial entre COVID-19 y otras enfermedades pulmonares agudas.

\section{VENTAJAS Y DESVENTAJAS}

Es una herramienta que puede utilizarse a la cabecera del paciente de manera rápida, evitando los desplazamientos de los pacientes necesarios para otras pruebas de imagen como la tomografía computarizada y, por lo tanto, limitando la exposición del personal. En este sentido, se ha propuesto que el uso del estetoscopio debe limitarse porque supone el contacto directo con el médico al colocarse directamente sin protección. ${ }^{11,12}$

El USP, sobre todo en su versión inalámbrica y portátil, puede cubrirse con una bolsa y debido a su pequeño tamaño se puede esterilizar por completo. Además, es una técnica segura para utilizar en grupos poblacionales específicos, como las embarazadas, en los cuales los métodos de imagen que utilizan radiación supondrían un riesgo.

Las desventajas que tiene este método es que, como toda técnica ecográfica, puede tener variabilidad entre los usuarios, además de que requiere un entrenamiento, el cual por la situación pandémica en la que vivimos no es fácilmente reproducible hoy en día. Por otra parte, se debe tener disponibilidad del equipo, lo cual no siempre está presente.

\section{PROPUESTA DE USO DE ULTRASONIDO PULMONAR}

Los expertos abogan por el uso de esta técnica en diferentes contextos durante la pandemia por SARS-CoV-2:

- En el diagnóstico en urgencias y el triaje de los pacientes que pueden ser dados de alta a domicilio.

- En el seguimiento en el paciente hospitalizado como predictor de empeoramiento respiratorio y necesidad de manejo avanzado de la vía aérea.

- En la monitorización de las terapias utilizadas y como ayuda en el proceso de destete del ventilador en pacientes intubados. 
Figura 2:

Ejemplo de un
protocolo de
adquisición de seis
sitios por hemitórax.
La importancia
de seguir un
protocolo ordenado
es esencial.

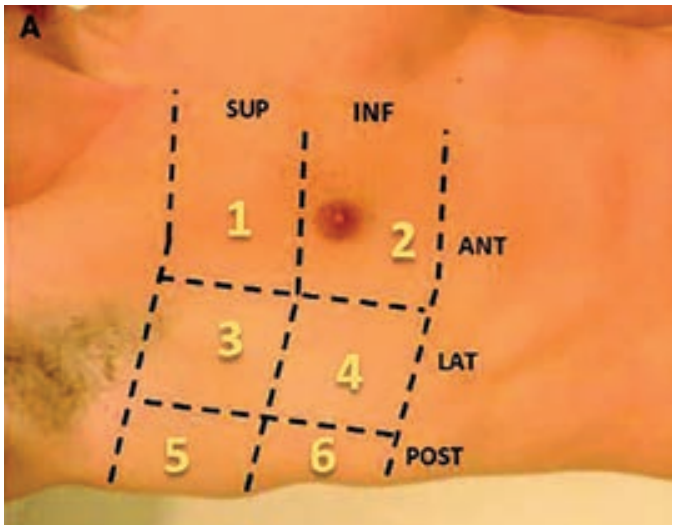

- Como parámetro de evaluación para un alta segura.

Sin embargo, en la actualidad, la evidencia disponible es escasa y uno de los principales retos que se presentan es la estandarización de la técnica para que los resultados de los diferentes grupos sean comparables.

Ante esta situación, recientemente se creó un sistema de puntuación para poder evaluar de manera objetiva la lesión pulmonar ocasionada por la COVID-19. Además, los artefactos ultrasonográficos pueden utilizarse para medir los efectos del reclutamiento pulmonar y para realizar pruebas con el fin de efectuar el destete ventilatorio. La propuesta de esta puntuación se basa en la revisión de seis áreas por hemitórax (12 zonas en total), donde cada una de las zonas puede recibir una puntuación que va desde 0 hasta tres. La definición de la puntuación se puede realizar con esta escala: a) patrón normal (presencia de líneas A y menos de tres líneas B), lo cual equivale a 0 puntos; b) más de tres líneas $B$, que equivale a un punto; c) líneas B coalescentes con o sin consolidaciones pequeñas, lo cual equivale a dos puntos, y d) consolidación, que equivale a tres puntos (Figura 2). Cabe recalcar que las puntuaciones de cada uno de los 12 puntos se suman y al final se puede obtener una puntuación que va desde 0 hasta $36 .^{7}$ Debido a la rápida evolución de la pandemia, este sistema de puntuación aún no ha sido validado; sin embargo, su aplicación posiblemente nos permitirá conocer de mejor manera la evolución de la enfermedad y también su progresión o regresión. ${ }^{12}$

\section{CONCLUSIÓN}

El USP es una herramienta fácilmente reproducible y que puede tener un papel muy importante en el diagnóstico y seguimiento de los pacientes con neumonía por SARS-CoV-2 en los lugares donde el equipo esté disponible y el personal tenga entrenamiento previo.

\section{REFERENCIAS}

1. Wu Y, Ho W, Huang Y, Jin DY, Li S, Liu SL et al. SARS$\mathrm{CoV}-2$ is an appropriate name for the new coronavirus. Lancet. 2020; 395 (10228): 949-950.

2. Guan WJ, Ni ZY, Hu Y, Liang WH, Ou CQ, He JX et al. Clinical characteristics of coronavirus dis-ease 2019 in China. N Engl J Med. 2020; 382: 1708-1720.

3. Tuite AR, Ng C, Rees E, Fisman D. Estimation of COVID-19 outbreak size in Italy. Lancet Infect Dis. 2020; 20 (5): 537.

4. Lichtenstein DA. The probe. In: Lichtenstein DA. Lung ultrasound in the critically ill. The BLUE Protocol. Heidelberg, Alemania: Springer; 2016. pp. 23-35.

5. Lichtenstein D, Méziére G. Relevance of lung ultrasound in the diagnosis of acute respiratory failure: the BLUE protocol. Chest. 2008; 134: 117-125.

6. Gopar-Nieto R, Alanís-Estrada GP, Ronquillo-Ramírez DE, Vargas-Estrada JL, Arias-Mendoza A, Rojas-Velasco $G$ et al. El ultrasonido pulmonar en cardiología: realidades y promesas. Arch Cardiol Mex. 2019; 89: 369-375.

7. Poggiali E, Dacrema A, Bastoni D, Tinelli V, Demichele E, Mateo-Ramos P et al. Can Lung US help critical care clinicians in the early diagnosis of novel coronavirus (COVID-19) pneumonia? Radiology. 2020: 295 (3) E6.

8. Soldati G, Smargiassi A, Inchingolo R, Buonsenso D, Perrone T, Briganti DF et al. Is there a role for lung ultrasound during the COVID-19 pandemic? J Ultrasound Med. 2020. 10.1002/jum.15284. [Epub ahead of print]

9. Smith MJ, Hayward SA, Innes SM, Miller ASC. Pointof-care lung ultrasound in patients with COVID-19 - a narrative review [published online ahead of print, 2020 Apr 10]. Anaesthesia. 2020. doi: 10.1111/ anae.15082.

10. Moro F, Buonsenso D, Moruzzi MC, Inchingolo R, Smargiassi A, Demi L et al. How to perform lung ultrasound in pregnant women with suspected $\mathrm{CO}$ VID-19. Ultrasound Obstet Gynecol. 2020; 55 (5): 593-598.

11. Kalafat E, Yaprak E, Cinar G, Varli B, Ozisik S, Uzun $C$ et al. Lung ultrasound and computed tomographic findings in pregnant woman with COVID-19. Ultrasound Obstet Gynecol. 2020; 55 (6): 835-837. doi: 10.1002/uog.22034.

12. van Doremalen N, Bushmaker T, Morris DH et al. Aerosol and surface stability of SARS-CoV-2 as compared with SARS-CoV-1. N Engl J Med. 2020; 382 (16): 1564-1567. doi: 10.1056/NEJMc2004973. 
13. Buonsenso D, Pata D, Chiaretti A. COVID-19 outbreak: less stethoscope, more ultrasound. Lancet Respir Med. 2020; 8 (5): e27. doi: 10.1016/S22132600(20)30120-X.

14. Buonsenso D, Piano A, Raffaelli F, Bonadia N, de Gaetano Donati K, Franceschi F. Point-of-care lung ultrasound findings in novel coronavirus disease-19 pneumoniae: a case report and potential applications during COVID-19 outbreak. Eur Rev Med Pharmacol Sci. 2020; 24: 2776-2780.

Correspondencia:

Diego Araiza-Garaygordobil

Juan Badiano Núm. 1,

Col. Belisario Domínguez,

Sección XVI, Tlalpan, 14080,

Ciudad de México, México.

E-mail: dargaray@gmail.com

\section{RESPONSABILIDADES ÉTICAS}

Protección de personas y animales. LoS autores declaran que para esta investigación no se han realizado experimentos en seres humanos ni en animales.

Confidencialidad de los datos. Los autores declaran que han seguido los protocolos de su centro de trabajo sobre la publicación de datos de pacientes.

Derecho a la privacidad y consentimiento informado. Los autores han obtenido el consentimiento informado de los pacientes y/o sujetos referidos en el artículo. Este documento obra en poder del autor de correspondencia. 